\title{
Du débat d'idées à la prise de pouvoir
}

Savoir et argumentation dans Bouvard et Pécuchet

\section{Florence Pellegrini}

\section{(CpenEdition}

Journals

Édition électronique

URL : http://journals.openedition.org/aes/584

DOI : $10.4000 /$ aes.584

ISSN : 2258-093X

Éditeur

Laboratoire LISAA

Référence électronique

Florence Pellegrini, « Du débat d'idées à la prise de pouvoir », Arts et Savoirs [En ligne], 1 | 2012, mis en ligne le 15 février 2012, consulté le 19 avril 2019. URL : http://journals.openedition.org/aes/584 ; DOI : 10.4000/aes.584

Ce document a été généré automatiquement le 19 avril 2019.

Centre de recherche LISAA (Littératures SAvoirs et Arts) 


\title{
Du débat d'idées à la prise de pouvoir
}

\author{
Savoir et argumentation dans Bouvard et Pécuchet
}

Florence Pellegrini

Cet article est la version remaniée d'une communication proposée dans le cadre du Colloque «Les Mots stratégiques dans le discours ", organisé à l'université de Bretagne Occidentale les 24 et 25 avril 2009 par le groupe de recherche "Mots de la controverse et déconstruction des discours " (EA 4249 HCTI, " Héritage et Constructions dans le Texte et l'Image »). Je remercie Ghislaine Lozachmeur de me permettre de présenter ce texte dans le cadre de la revue Arts et Savoirs.

Dans « l'encyclopédie critique en farce » ${ }^{1}$ qu'est Bouvard et Pécuchet ${ }^{2}$, le débat d'idées tient une place fondamentale, basilaire: la construction en miroir des protagonistes, qui littéralement incarnent les idéologies antinomiques et les savoirs contradictoires qu'ils lisent, permet un jeu sur la symétrie et l'opposition qui est une constante de l'œuvre. La caractérisation des protagonistes en exacte antithèse, qui « pli[e] en matière romanesque le débat [scientifique et] philosophique $»^{3}$, consent une confrontation des postures bien souvent résorbée dans l'aporie identitaire ${ }^{4}$. Les distensions conceptuelles s'annihilent dans la logique du « rien ne vaut » telle que l'a définie Philippe Dufour dans son Essai sur la représentation romanesque du langage $e^{5}$, logique nivelante qui réduit au même les disparités. Le fonctionnement du chapitre viII, qui condense la matière philosophique, est à ce titre emblématique: le débat engagé dans la symétrie inversée des postures idéologiques, depuis les origines - » Aristote » vs «Platon »- jusqu'aux représentants de la "philosophie moderne", présente, dans le pêle-mêle de l'énumération, une historiographie confuse de la pensée qui achoppe sur une conclusion lapidaire : « tant de systèmes vous embrouille. La métaphysique ne sert à rien. On peut vivre sans elle. » Ainsi évacué sinon résolu, le débat est réorienté vers une autre discipline, relancé dans un autre domaine, ajoutant un tour supplémentaire à la spirale sapientiale qui constitue la structure portante du roman ${ }^{6}$. De cette tension essentielle au sein du binôme, le récit tire une dynamique, qui lui permet de ne pas sombrer dans la " dissertation philosophique $»^{7}$ mais bien de conserver une forme de progression narrative minimale qui fait passer les bonshommes de l'agriculture (chapitre II) à l'éducation (chapitre $\mathrm{x}$ ), après avoir traversé 
des champs de savoir au statut épistémologique divers : chimie, médecine, géologie (III) ; archéologie, histoire (IV); littérature (v); politique (VI); amour (VII); occultisme, philosophie (VIII) ; religion (Ix) ${ }^{8}$. Les divergences d'opinion, tout comme les différences de caractérisation entre Bouvard - » aimable », souriant, " gros et gras, le teint frais et [le visage sinon la bouche] vermei[1] " - et Pécuchet, « sérieux », " caverneux », «tout en profil » (48) - sont fonctionnelles : elles consentent la création d'un espace de discussion, souvent animé, parfois polémique, qui fait s'entrechoquer, à travers les discours de savoir qu'il confronte, des représentations contradictoires du réel.

2 Le phénomène s'amplifie lorsque les compères se trouvent en présence des habitants du petit bourg de province où ils ont élu domicile : les joutes verbales qui les opposent aux notables chavignollais répliquent en la démultipliant cette dualité antagoniste initiale. De la même façon qu'Emma, dans Madame Bovary, s'oppose à la médiocrité bourgeoise des habitants de Yonville, Bouvard et Pécuchet, ces « Ulysse du savoir », comme les a désignés Raymond Queneau, affrontent les notables de leur village. Ce sont des conflits permanents entre les deux amis et les Chavignollais qu'ils écrasent de leur mépris :

- Quel pays! on n'était pas plus inepte, sauvage et rétrograde! La comparaison qu'ils firent d'eux-mêmes avec les autres les consola. (110)

- Alors une faculté pitoyable se développa dans leur esprit, celle de voir la bêtise et de ne plus la tolérer. [...] En songeant à ce qu'on disait dans leur village, et qu'il y avait jusqu'aux antipodes d'autres Coulon, d'autres Marescot, d'autres Foureau, ils sentaient peser sur eux comme la lourdeur de toute la terre. (298)

3 Ainsi, le roman pourrait bien n'être qu'une succession d'épisodes conflictuels où s'affrontent théories et opinions. Ou plutôt théories réduites en opinions, schémas hérités, idées reçues, paroles aussi catégoriques que figées et qui finalement s'équivalent, dans une égale et vaine présomption; l'hostilité de la communauté chavignollaise n'a d'égale que l'obstination des bonshommes à défendre leur position, dans une attitude opiniâtre où la posture d'adhésion compte davantage que le savoir auquel on adhère. Car ce qui est en jeu dans la confrontation des opinions, c'est bien l'ascendant qu'il s'agit de prendre sur l'interlocuteur, partant le statut social que l'on est à même d'assumer. Dans un contexte sinon toujours fortement polémique - c'est le cas des grands moments dialogués du roman qui voient Bouvard et Pécuchet se heurter à la respectabilité aussi conformiste que dogmatique des habitants de Chavignolles - du moins de nécessaire confrontation, les stratégies argumentatives mises en œuvre par les personnages modulent les énoncés d'une intentionnalité inavouée d'écrasement de l'adversaire, de sa réduction au silence et cela indépendamment du sujet abordé. Le partage des savoirs touche ainsi au politique: moins enjeu scientifique que rapport de forces, la représentation des disciplines est, dans Bouvard et Pécuchet, lieu de prépondérance et d'autorité. C'est cette évacuation du débat d'idées au profit d'une rhétorique oppressive destinée à asseoir la prévalence du locuteur que cet article entend explorer : à travers l'utilisation de connecteurs argumentatifs récurrents, pivots des argumentations déployées et manifestes dans les discours des protagonistes comme dans ceux des personnages secondaires, se dessine une logique d'oppression, la question épistémologique étant étroitement corrélée à celle du pouvoir. Dans un gauchissement des chaînes de raisonnement se lit alors la prétention autoritaire des discours assénés et, en arrière-plan, une réflexion sur le désir de connaissance, qui, dans sa forme dévoyée mais ô combien commune, est aussi et surtout une volonté de puissance.

4 L'indifférenciation terminologique - tous les personnages usent des mêmes tours et des mêmes connecteurs -, le flottement énonciatif - l'origine des énoncés est souvent 
incertaine -, l'absence de spécification discursive - pas de pittoresque langagier ou d'idiolecte caractérisant et typisant un personnage-, rabattent le dialogique sur le monologique : les discours ne ménagent aucune place à l'interlocuteur et sont autant de soliloques juxtaposés qui déploient une stratégie offensive qui se veut bien souvent moins persuasive qu'écrasante.

Je me propose ainsi d'étudier trois moments de cette stratégie, chacun articulé autour d'un joncteur ou d'un embrayeur de discours qui exclut toute réplique : d'ailleurs et la reconstruction a posteriori d'un argumentaire absent qu'il autorise - cette "logique du camelot » telle que l'a définie Oswald Ducrot ${ }^{10}$; mais qui dessine une rhétorique du viceversa et finalement bloque la chaîne argumentaire; n'importe et qu'importe, indices tendanciels de cette fin de non-recevoir secrètement larvée dans les propos de chacun des acteurs du débat.

6 Une remarque préliminaire : la distinction entre récit et discours est problématique chez Flaubert en général, dans Bouvard et Pécuchet en particulier. Les travaux consacrés à l'analyse, dans la prose flaubertienne, de ce que Ducrot désigne en termes de polyphonie linguistique ou d'un dialogisme, au sens bakhtinien d'une hétérogénéité constitutive du langage, s'accordent à souligner la contamination discursive du narratif, contamination dont la prégnance si caractéristique du discours indirect libre serait la manifestation la plus symptomatique. De fait, la frontière entre les différentes modalités énonciatives s'estompe, rendant définitivement incertaine sinon totalement inopérante toute tentative de catégorisation traditionnelle. En ce qui concerne l'inclusion dans le récit de la parole des personnages, la fiction expérimente des procédés de brouillage des instances énonciatives et d'indétermination qui contreviennent à la séparation entre narratif et discursif. Dans le cadre de cet article, je m'attacherai préférentiellement aux joncteurs en discours direct, tout en précisant que les stratégies discursives identifiées sont également celles qui régissent l'organisation diégétique et la structure narrative.

7 Fausse logique conclusive, rétroversion de l'argumentation, « coup de force $\mathrm{d}[\mathrm{e}$ la] parole intolérante $»^{11}$ ou évitement: ces trois modalités argumentatives complémentaires soulignent moins l'impossibilité de la communication entre les individus - c'est le " chaudron fêlé » de la parole humaine théorisé dans Madame Bovary - qu'elles ne révèlent la volonté oppressive de chaque locuteur. Les échanges de paroles entre personnages s'affirment comme un jeu de pouvoirs où le point de vue asserté compte moins, en définitive, que l'assertion elle-même, l'énonciation devenant le garant de l'ascendant pris sur l'interlocuteur, ou mieux, d'une suprématie discursive.

\section{Détourner la discussion : l'art du boniment}

8 L'analyse qu'Oswald Ducrot propose du connecteur d'ailleurs ${ }^{12}$ insiste d'une part sur sa valeur argumentative, d'autre part sur son impossibilité d'emploi en début d'énoncé : "l'énoncé [...] régi par d'ailleurs présente toujours un argument [non nécessaire] qui s'ajoute à un argument ou à un ensemble d'arguments antérieurs. $»^{13}$ Le repérage de d'ailleurs permet une construction rétrospective de la logique du texte. L'énoncé qui précède se trouve valorisé, acquiert une cohérence démonstrative qu'il n'aurait pas nécessairement sans sa présence : «dans la structure $P$ d'ailleurs $Q, P$ reçoit une valeur argumentative qu'il n'aurait pas si $Q$ lui était simplement ajouté, ce qui pourrait laisser entendre que $P$ ne suffit pas. $»^{14} C^{\prime}$ est ce que Ducrot nomme la «logique du camelot»: 
l'argument introduit par d'ailleurs étant facultatif, il n'a pas à être justifié ; inversement, la présence de d'ailleurs garantit la validité argumentative de l'énoncé qui le précède.

9 Ainsi au chapitre II, lorsque le comte de Faverges s'attache à convaincre Bouvard et Pécuchet, néo-ruraux béotiens, du bien fondé de ses méthodes agricoles :

$\mathrm{Vu}$ le nombre de ses bestiaux, il s'appliquait aux prairies artificielles: c'était d'ailleurs un bon précédent pour les autres récoltes, ce qui n'a pas toujours lieu avec les racines fourragères. - » Cela du moins me parait incontestable. »

Bouvard et Pécuchet reprirent ensemble : «Oh ! incontestable. » (72)

10 La dimension discursive du connecteur apparaît nettement dans l'exemple qui associe deux formes de discours rapporté : la démonstration du comte de Faverges, au style indirect libre, trouve sa conclusion dans l'assertion catégorique au style direct que répètent docilement les compères. Quelle que soit la qualité intrinsèque du premier argument avancé par le comte pour justifier ses "prairies artificielles", son raisonnement se trouve validé par l'ajout in extremis d'un argument superfétatoire. Les deux arguments sont autonomes, indépendants sur le plan sémantique comme sur le plan logique, et l'argument final est donné dans un «second mouvement discursif », c'est-àdire que le locuteur « se présente comme ayant d'abord voulu donner le seul argument $P$, et comme donnant l'argument $Q$ après coup. $»^{15}$ Dans la mesure où le second argument n'est pas indispensable, il n'a pas à être justifié ; il devient par là même « incontestable ». Et la certitude finale se répercute sur l'ensemble de la discussion. L'importance du bétail devient un argument pertinent pour expliquer les «prairies artificielles » parce que ces dernières sont bénéfiques pour les « autres récoltes » et que cela n'a pas à être démontré. On ne peut alors qu'en conclure le savoir-faire du comte ${ }^{16}$. Et les deux bonshommes d'acquiescer.

11 D'ailleurs opère un véritable détournement qui clôt l'argumentation en l'éloignant $d u$ sujet initial. Le connecteur instaure une logique de la digression longuement étudiée par Philippe Dufour à propos des discours du pharmacien de Madame Bovary:

la rhétorique d'Homais est rhétorique de l'amplification. Le point final de la phrase, le terme de la réplique sont différés par de constants ajouts qui permettent à Homais de monopoliser la parole et transforment ses interventions en tirade. [...] Le pharmacien affectionne particulièrement des adverbes comme d'ailleurs, du reste, petits embrayeurs de digression qui lui laissent orienter la conversation et repoussent à volonté la clausule du discours. ${ }^{17}$

Mais l'utilisation du connecteur indique également un discours autoritaire. D'ailleurs introduit

souvent une loi. Derrière l'appétit de parole d'Homais, derrière sa phrase centrifuge dont les réticulations témoignent d'un désir de traiter tous les sujets, de traiter un sujet sous tous ses aspects, se découvre un univers de la surdétermination. Homais n'est jamais en mal de raisons. Le d'ailleurs soutient qu'il peut toujours en fournir de nouvelles; l'adverbe d'ailleurs représente l'exaltation du principe de raison suffisante, son hyperbolisation. ${ }^{18}$

C'est bien ce qui se passe dans le discours du comte de Faverges qui, fort de son expérience en matière d'agriculture et de sa position de modèle, n'entend pas être remis en cause par les nouveaux arrivants.

Il n'est pas surprenant dans ces conditions de voir apparaitre le connecteur dans les propos de l'abbé Jeufroy ou du docteur Vaucorbeil. Garants respectifs de l'orthodoxie religieuse et médicale, ils développent une parole dogmatique, qui assène et sermonne sans avoir à se justifier. 
1- Devant ce blasphème le curé pâlit. Ses prunelles fulguraient. Sa mâchoire tremblait. - « Taisez-vous, malheureux ! [Il s'adresse à Petit, l'instituteur] Taisezvous!»

«Et c'est sa femme qui soigne les linges de l'église !»

- « Eh bien? quoi ? qu'a-t-elle fait?»

- « Elle manque toujours la messe ! - Comme vous, d'ailleurs !» (231)

2- « Moi j'admets un fluide » reprit Bouvard.

- « Nervoso-sidéral » ajouta Pécuchet.

- « Mais prouvez-le ! montrez-le ! votre fluide ! D’ailleurs les fluides sont démodés :

écoutez-moi. » (270)

14 Les griefs du curé envers l'instituteur se transmettent à son épouse avant que de se transformer en reproches à l'égard de Pécuchet (1). Par cette condamnation généralisée, Jeufroy interdit à quiconque de contester son autorité. Le droit de regard qu'il s'arroge sur les contenus d'enseignement de Petit, avalisé par la loi (« je vous rappelle que la loi du 15 mars nous attribue la surveillance de l'instruction primaire »), n'a pas à être remis en cause, ni même discuté. Si l'instituteur, de fait, doit s'incliner, il en est de même pour Pécuchet dont la véhémence est court-circuitée par l'attaque finale de Jeufroy.

Même chose dans le débat qui oppose Bouvard et Pécuchet magnétiseurs à Vaucorbeil : la discussion sur le «fluide » est évacuée avant même que d'avoir eu lieu. Les objections du médecin (« mais ») frappent par leur ton agressif, menaçant (« Mais prouvez-le ! montrezle!»). Le défi lancé aux compères (réitération de tours impératifs) vaut pour toute argumentation. Et la réflexion achoppe sur une clausule catégorique (d'ailleurs suivi d'une assertion au présent gnomique) : l'affirmation du caractère obsolète du fluide se substitue à la démonstration de son existence. L'énervement de Vaucorbeil, manifeste dans l'utilisation répétée de la modalité exclamative, tend à se dissimuler derrière la fausse logique de son raisonnement. La parole émise par le médecin a valeur de loi et ne tolère, pas plus que celle du curé, la contradiction.

Ainsi la clôture de l'énoncé par d'ailleurs restaure la cohérence argumentative du passage qui précède le connecteur, ou plutôt souligne l'illusion de cette cohérence. Car l'on peut aussi dire que l'argument final en porte-à-faux décrédibilise l'ensemble du raisonnement : la justification supplémentaire introduite par d'ailleurs suggère la faille, le gauchissement logique, le boniment. S'il est toujours possible d'ajouter une raison supplémentaire, il n'en est aucune qui vaille. Aucune n'est en soi suffisante pour garantir la stabilité du texte ; la dernière cause arguée n'est même pas nécessaire. C'est l'accumulation, la liste qui tient lieu d'argumentaire: la syntaxe de l'ajout se substitue à une argumentation défaillante.

\section{Arrêter la discussion : thèse, antithèse... sans synthèse}

17 La faille - et parfois la faillite - du raisonnement est également perceptible dans l'utilisation de la coordination mais, qui non seulement bloque la chaîne argumentative par l'intrusion d'une objection mais réduit à néant, par le mouvement de retour en arrière qu'elle opère, l'enchaînement discursif qui la précède, ce que Ducrot désigne comme le renversement de l'orientation argumentative du propos. La discussion piétine, dans un mouvement de va-et-vient argumentatif qui l'immobilise. 
ne s'agit pas d'opposer le contenu des propositions que la conjonction coordonne mais bien «la conclusion vers laquelle ces propositions pourraient mener $»^{19}$. Ducrot, on le sait, distingue «deux mais en français $»^{20}$ : d'une part le mais d'opposition, qui permet d'opposer le contenu sémantique de deux énoncés ; d'autre part le mais argumentatif ou de réfutation, qui oppose une seconde proposition non pas à la première mais aux conclusions que l'on pourrait en tirer. Anne Herschberg Pierrot a souligné la prépondérance du mais argumentatif dans Madame Bovary ${ }^{21}$. C'est également le cas dans Bouvard et Pécuchet, où la fréquence tout à fait remarquable du connecteur - en moyenne une occurrence par page - relève de cette logique des « deux colonnes » mise en évidence par Juliette Grange ${ }^{22}$ : mais vient interrompre un enchaînement argumentatif, lui met un terme et, simultanément, le réoriente en sens inverse, rabattant la progression discursive sur une bipolarité stagnante.

Intégré au discours des personnages, mais manifeste un affrontement insoluble, une confrontation stérile d'opinions, dans le renversement des postures en exacte antithèse. Il n'y a donc jamais d'évolution, de progression dans le débat d'idées mais la substitution, l'évacuation d'une opinion par son contraire. Révélatrices de ce système autoritaire et vindicatif du "pousse-toi que je m'y mette», les discussions animées entre les personnages qui ne génèrent qu'une cacophonie généralisée où s'impose non le jugement le plus pertinent - car tous se valent dans un tel dispositif textuel - mais la loi de celui qui parle le plus fort.

Au chapitre III, les études géologiques de Bouvard et Pécuchet les amènent à interroger l'abbé Jeufroy «sur le Déluge ». À la discussion entre les compères et l'ecclésiastique se joignent progressivement «le maire», «M. Girbal, directeur des Contributions», «le capitaine Hertaux, propriétaire », Beljambe, l'aubergiste, l'épicier Langlois, enfin le comte de Faverges, dernier arrivé pour une réunion du «Conseil de fabrique» au sujet de «l'acquisition d'une chasuble».

Que signifiait dans la Genèse, « l'abîme qui se rompit » et « les cataractes du ciel »? Car un abîme ne se rompt pas, et le ciel n'a point de cataractes !

L'abbé ferma les paupières, puis répondit qu'il fallait distinguer toujours entre le sens et la lettre. Des choses qui d'abord nous choquent deviennent légitimes en les approfondissant.

- «Très bien! mais comment expliquer la pluie qui dépassait les plus hautes montagnes, lesquelles mesurent deux lieues! y pensez-vous, deux lieues! une épaisseur d'eau ayant deux lieues! »

Et le maire, survenant, ajouta : - «Saprelotte, quel bain!»

- «Convenez » dit Bouvard « que Moïse exagère diablement. »

Le curé avait lu Bonald, et répliqua : - «J'ignore ses motifs ; c'était, sans doute, pour imprimer un effroi salutaire aux peuples qu'il dirigeait !»

- «Enfin, cette masse d'eau, d'où venait-elle?»

- «Que sais-je ? L'air s'était changé en pluie, comme il arrive tous les jours.» [...]

- «Mais si votre Déluge» repartit Bouvard «avait charrié des coquilles, on les trouverait brisées à la surface, et non à des profondeurs de trois cents mètres quelquefois. »

Le prêtre se rejeta sur la véracité des Écritures, la tradition du genre humain et les animaux découverts dans la glace, en Sibérie. [...]

Comme les allées étaient trop petites, en gesticulant, on marchait dans les platesbandes. Langlois fut pris d'une quinte de toux. Le capitaine criait : «Vous êtes des révolutionnaires! » Girbal : «La paix ! la paix ! » Le prêtre : «Quel matérialisme !» Foureau : « Occupons-nous plutôt de notre chasuble !»

- «Hou! Laissez-moi parler!» Et Bouvard s'échauffant, alla jusqu'à dire que l'Homme descendait du Singe ! 
Tous les fabriciens se regardèrent, fort ébahis, et comme pour s'assurer qu'ils n'étaient pas des singes.

Bouvard reprit: - «En comparant le fœtus d'une femme, d'une chienne, d'un oiseau..."

- «Assez!»

- «Moi, je vais plus loin!» s'écria Pécuchet. «L'homme descend des poissons!»

Des rires éclatèrent. Mais sans se troubler : «Le Telliamed ! un livre arabe... »

- «Allons, messieurs, en séance !» (143-146)

21 souhaite que réduire l'autre au silence. Les injonctions finales de Girbal («La paix! la paix!») ou de Foureau (« Occupons-nous plutôt de notre chasuble!») visent à abréger l'échange et à faire taire un Bouvard " échauff[é] ", contraint à l'onomatopée pour attirer l'attention et récupérer ainsi la parole («Hou! Laissez-moi parler!»). Les objections répétées interdisent le développement de la réflexion; la logique semble absente d'un débat où aucun des personnages ne ménage une place à ses interlocuteurs. Plus que d'un véritable dialogue, nous sommes en présence d'une accumulation de monologues parfaitement hermétiques et restreints à la plus primaire affectivité, qui font succéder les exclamations aux cris. Les verbes déclaratifs sont pour la plupart des verbes affectifs à connotation agressive : "répliquer ", "répartir ", "crier ", "s'écrier » indiquent une surenchère dans la riposte, un haussement du ton. Philippe Dufour a souligné la "violence stichomythique [de ce] langage ${ }^{23}$ où ce qui est dit compte moins que le ton sur lequel on s'exprime :

Le verbe déclaratif flaubertien [et la modalité phrastique sont] l'équivalent romanesque des propos de La Bruyère : «Dire d'une chose modestement ou qu'elle est bonne ou qu'elle est mauvaise, et les raisons pourquoi elle est telle, demande du bon sens et de l'expression. Il est plus court de prononcer d'un ton décisif et qui emporte la preuve de ce qu'on dit, qu'elle est exécrable ou qu'elle est miraculeuse. » 24

Ce que la lecture retient de ce type de dialogue, c'est la véhémence d'une parole intolérante, dont le sens se perd dans l'inachèvement et l'indétermination énonciative : les derniers propos de Pécuchet restent en suspens, à jamais incomplets et incompréhensibles, alors que ses interlocuteurs, unis dans une injonction non originée - »Assez » et «Allons, messieurs »-, le réduisent définitivement au silence par leur départ.

Il serait rassurant de voir, dans cette objection tenace qui émerge sans cesse par l'intermédiaire du mais la marque d'une perturbation salutaire : comme le note Philippe

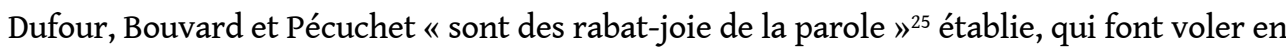
éclats, par leurs critiques et leurs remises en cause, les évidences trop nettes des discours dominants et perturbent le déroulement serein d'une parole consensuelle dans laquelle peuvent se reconnaître les autres personnages. Certes, il y a de cela, et les antagonismes massifs entre les protagonistes et les notables du village dénotent cette perturbation radicale instillée au cœur de la doxa. Mais, dans la mesure où Bouvard et Pécuchet incarnent à leur tour des paroles autoritaires susceptibles de s'affronter, qu'il s'agisse d'un affrontement entre les deux bonshommes ou d'une distension entre deux postures successives d'un même personnage, c'est la possibilité même d'une parole harmonieusement déployée qui est réduite à néant.

À l'intérieur de ces conflits scandés par mais, tout raisonnement élaboré est exclu: chacun s'attache à trouver un intervalle où il pourra exhaler son indignation et s'ingénie à interrompre l'autre. Ainsi sous le " poirier de Puységur », au chapitre viII :

Arts et Savoirs, 1 | 2012 
Beljambe avait rêvé, bien des fois, le nombre de voyageurs qu'il aurait le lendemain à son auberge. Et Petit conta le souper de Cazotte.

Le curé, alors, fit cette réflexion: - «Pourquoi ne pas voir là dedans, tout simplement...

- « Les démons, n'est-ce pas?» dit Vaucorbeil.

Marescot parla de la Pythie de Delphes.

- «Sans aucun doute, des miasmes... »

- «Ah! les miasmes, maintenant!»

- « Moi, j'admets un fluide » reprit Bouvard.

- « Nervoso-sidéral » ajouta Pécuchet.

- «Mais prouvez-le ! montrez-le ! votre fluide ! D’ailleurs les fluides sont démodés ;

écoutez-moi. » (270)

\section{indifféremment utilisées - marque le caractère inopérant de l'argumentaire par rapport à} l'énoncé prééminent que la locution introduit et qui vient clore le débat.

Au chapitre III, la typhoïde de Gouy, le fermier de Bouvard et Pécuchet, est l'occasion d'un affrontement théorique entre Pécuchet et le médecin Vaucorbeil, qui vise moins à la guérison du malade qu'à l'établissement de la suprématie d'un personnage ${ }^{27}$. L'argumentaire déployé par Vaucorbeil à grand renfort de liens logiques (« Vous perforez l'intestin, puisque la fièvre typhoïde est une altération de sa membrane folliculaire.», «Aussi j'éloigne tout ce qui peut surexciter!», «D’ailleurs Gouy ne veut pas de nourriture. ») est destiné à neutraliser les velléités médicales de Pécuchet qui menacent "son gagne-pain» et "son importance sociale» (122). Pourtant, et en dépit de la légitimité de sa parole, étayée d'autorités arguées, le médecin n'aura pas le dernier mot et ses tentatives de redéploiement du débat tourneront court.

Tout à coup, le docteur parut.

Son malade était en train de manger, deux oreillers derrière le dos, entre la

fermière et Pécuchet qui le réforçaient.

Il s'approcha du lit, et jeta l'assiette par la fenêtre, en s'écriant :

- «C'est un véritable meurtre!»

- «Pourquoi?»

- «Vous perforez l'intestin, puisque la fièvre typhoïde est une altération de sa membrane folliculaire. » 
- «Pas toujours!»

Et une dispute s'engagea sur la nature des fièvres. Pécuchet croyait à leur essence. Vaucorbeil les faisait dépendre des organes : - «Aussi, j'éloigne tout ce qui peut surexciter!»

- « Mais la diète affaiblit le principe vital!»

- «Qu'est-ce que vous me chantez avec votre principe vital ? Comment est-il ? Qui

l'a vu?»

Pécuchet s'embrouilla.

- «D'ailleurs » disait le médecin, « Gouy ne veut pas de nourriture. »

Le malade fit un geste d'assentiment sous son bonnet de coton.

- « N'importe! il en a besoin!»

- «Jamais! son pouls donne quatre-vingt-dix-huit pulsations. »

- «Qu'importe les pulsations ! " Et Pécuchet nomma ses autorités.

- « Laissons les systèmes ! » dit le docteur. (121) Vaucorbeil à ses accusations (« c'est un véritable meurtre »; « vous perforez l'intestin »), pour être catégorique - on notera l'utilisation du présent gnomique et le tour généralisant -, n'en est pas moins douteuse. Introduite par le biais du connecteur polyphonique puisque, elle vaut moins par son contenu que par son énonciation. Je m'appuierai une nouvelle fois sur la description du connecteur proposée par Oswald Ducrot $^{28}$ : l'utilisation de puisque suggère que le locuteur-Vaucorbeil « fait s'exprimer un énonciateur dont il se déclare distinct [...]. Autrement dit, le locuteur ne s'engage pas sur [l'énoncé introduit par puisque], il n'en prend pas la responsabilité. $»^{29}$ Leçon apprise sans être assumée, le discours scientifique dont Vaucorbeil se veut le porte-parole se trouve alors dévalorisé. Parole héritée, dogmatique, reproduite sans être investie par le locuteur, la proposition introduite par puisque est la marque d'un conformisme psittacique qui la discrédite. La déduction qu'opère Vaucorbeil est rendue contestable - ou plutôt elle ne vaut que dans un système où l'on reconnaît et à Vaucorbeil et à ses références une valeur d'autorité. Or c'est précisément ce que Pécuchet refuse au médecin auquel il ambitionne de se substituer - et cette contestation se répercute sur les doctrines qui servent à la justifier. À l'interrogation causale de Pécuchet (« Pourquoi ?»), Vaucorbeil n'offre qu'une réponse décalée, qui ne porte pas véritablement sur la cause ( " puisque ») et devient par là même incertaine, comme le souligne la clausule du dialogue (« Pas toujours !»).

D'autre part, le recours final à l'adverbe d'ailleurs révèle une tentative d'échappatoire: c'est par un glissement de l'argumentaire, l'ajout in fine d'un argument superfétatoire que Vaucorbeil entend remporter la « dispute ». Mais Pécuchet écarte d'un mot - de deux, en fait - cette tentative : signes de répression et de prévention, n'importe et qu'importe font tourner court la discussion. "Élusif[s], coup[s] de force d'une parole intolérante, il[s] remet[tent] la conversation dans le droit chemin $»^{30}$ et réduisent à néant les objections. " N'importe, Qu'importe: les locutions exclamatives s'emboitent, symboles d'un discours où tout était décidé d'avance ; le diagnostic avait précédé l'auscultation. Avec le n'importe la parole prend de vitesse le réel, le devance sans plus se soucier de s'ajuster à lui. Elle affirme son indépendance (il ne s'agit pas même de convaincre l'autre; simplement de garder ses certitudes). $»^{31}$ Pécuchet entend «enfoncer Vaucorbeil» (266), non par la validité de sa démonstration médicale, mais bien par l'offensive du n'importe qui rend insignifiante la parole adverse. Et c'est bien lui qui aura gain de cause au final, obligeant Vaucorbeil, dénié dans sa « prérogative » (122), à battre en retraite :

31 [...] se tournant vers la fermière : « Faites-le tuer par monsieur tout à votre aise, et que je sois pendu si je reviens jamais dans votre maison!» 
Et il s'enfonça sous la hêtrée, en gesticulant avec sa canne. (122)

La question n'est bien évidemment pas de guérir le « somnolent » Gouy, qui, ne sachant à quelle parole autoritaire se vouer, se «m[et] à pleurer» de détresse et d'indécision. L'épisode se referme sur le départ de Vaucorbeil et si la "guérison» du fermier est ensuite affirmée comme "certaine » (123), ce n'est qu'après avoir évoqué les douleurs abdominales consécutives aux soins de Pécuchet, qui suffisent à instiller un doute quant à ses compétences.

Gouy, le lendemain, eut une douleur dans l'abdomen. Cela pouvait tenir à l'indigestion de nourriture? Peut-être que Vaucorbeil ne s'était pas trompé ? Un médecin, après tout, doit s'y connaître! Et des remords assaillirent Pécuchet. Il avait peur d'être homicide. (123)

On voit bien que ce qui est en jeu ici est de l'ordre de la prise de pouvoir et que les deux locuteurs opposés se rejoignent dans la symétrie des postures: au catégorique «Pas toujours ! » de Pécuchet répond le définitif «Jamais ! de Vaucorbeil. Et il est amusant de constater que c'est la " pratique », celle-là même que Vaucorbeil préconisait (« D’abord, il faut avoir fait de la pratique »,122) et que Pécuchet refusait («Ceux qui ont révolutionné la science, n'en faisaient pas ! »,122), qui assurera le « succès » du bonhomme. Aux doutes et aux questionnements du protagoniste - » après tout », il n'est pas médecin !- succède rapidement la satisfaction du résultat obtenu et ce sans que l'on sache véritablement à quoi il est dû. N'importe: la parole divergente, vecteur de doute, est rejetée par les personnages. Tous esquivent la contestation et, sous des antagonismes de surface, se rejoignent dans l'idéologie commune de la vérité assénée.

« Avec le n'importe [...] l'idéologie s'entête dans son illusion d'être le discours de la vérité. [...] La "vérité" prise en défaut par les faits se mue en une vérité de foi, un dogme. N'importe est la syntaxe du mystère. $\|^{32}$ C'est ce que suggère malicieusement la réaction de la fermière à la demande de Vaucorbeil qui l'exhorte de choisir entre lui et Pécuchet: « [elle] ne savait que répondre; car l'un [Vaucorbeil] était habile; mais l'autre [Pécuchet] avait peut-être un secret ?» (123)

35 À défaut d'argument d'autorité validé par les interlocuteurs, n'importe articule l'autorité, "met un point final au dialogue ou en tout cas le dévie, "détourne la conversation" comme si la réplique précédente n'existait pas. " $^{33}$ N'importe balaie négligemment les objections, proclamant l'inaltérabilité du discours de loi, qui est en premier lieu un discours de foi au sens où il est un discours d'adhésion. N'importe supprime la discussion, sacralise l'impensé, le principe hérité, c'est ce que stipule le Dictionnaire des Idées Reçues :

\section{PRINCIPES Toujours indiscutables}

On ne peut en dire ni la nature, ni le nombre

N'importe, sont sacrés. (433)

Il y a, bien sûr, une limite à cette rhétorique implacable. L'exemple du chapitre III le souligne : la clôture du dialogue par n'importe est sujette à caution. Et Vaucorbeil est un adversaire coriace qui n'entend pas cautionner, c'est-à-dire se laisser imposer par autrui un discours de loi dont il devrait être seul dépositaire. Mieux : le docteur sait récupérer la logique de déplacement introduite par n'importe. Son argumentaire en faveur de la diète de Gouy restant inopérant, il relève le pouls accéléré du fermier, preuve d'une autre nature destinée à réorienter et à relancer le débat. Pécuchet doit alors réitérer l'objection ( Qu'importe les pulsations!»), qu'il étaye de la mention de ses « autorités». Vaucorbeil doit alors battre en retraite, pris à son propre piège autoritaire: «laissons les systèmes ! », enjoint-il, défait. 
ourtant, la mention in fine des "autorités » marque bien toute l'ambiguïté - voire l'insuffisance - du n'importe et du qu'importe. Dans le même temps où ils évacuent la parole importune, ils

marque[nt] aussi la contradiction quand il[s] voudrai[ent] la masquer. [...] Il[s] porte [nt] en [eux leur] dénégation: le n'importe dit qu'il importe. Son apparente assurance laisse percer la vérité flaubertienne par excellence, la maxime de Flaubert - qui est une anti-maxime : il est impossible de conclure. ${ }^{34}$

Baratin, retournement stérile, sourde oreille et mauvaise foi: les stratégies argumentatives à l'œuvre dans Bouvard et Pécuchet ne laissent pas d'interroger. «Prose des savoirs ", comme l'a désignée Jacques Neefs ${ }^{35}$, la fiction flaubertienne met en scène leurs discours, dans un processus d'exposition qui est en même temps une remise en cause $^{36}$ :

le romanesque de Bouvard a été façonné à partir des cadres ou des articulations donnés par chaque science. La critique génétique a souvent insisté sur ce point. En fait, les rapports entre l'instance romanesque et l'instance épistémologique sont plutôt réciproques, car le roman flaubertien et son avant-texte ont une capacité particulière d'exposition. Ils nous montrent [l]es contradictions entre auteurs ou entre théories, [l]es enjeux idéologiques de[s] discours scientifiques [...]. ${ }^{37}$

La parole des personnages est le lieu d'exposition privilégié de ces antagonismes idéologiques qui structurent, sous-tendent, travaillent le récit. Conflit ouvert ou affrontement larvé, chaque discussion révèle le désir tyrannique de locuteurs qui visent non pas à convaincre mais à prendre l'ascendant. La contradiction a alors valeur d'un règlement de comptes. L'enjeu n'est pas le savoir, mais bien l'utilisation du savoir à des fins oppressives. Les certitudes s'alternent, dans un ping-pong verbal sans autre issue possible que la fuite ou la réduction au silence de l'interlocuteur.

41

Mais la prise de pouvoir est temporaire et réversible. Bouvard et Pécuchet, victorieux ici, seront défaits là et, tout comme eux, potentiellement, chacun des personnages, selon qu'il s'inscrit ou non dans un système de croyance dominant. Car c'est le degré d'adhésion qui fonde la légitimité de la parole et ce, indépendamment de son contenu. "Qu'importe la croyance! Le principal est de croire», finira par admettre Pécuchet. Qu'importe la parole! le principal est qu'elle ait force de loi. Et les discours de tourner, dans le maelström perpétuel des opinions irréconciliables et pourtant équivalentes d'autoritarisme. À défaut "d'attendrir les étoiles ", la parole humaine fera bien « danser les ours » dès lors qu'elle les aura domptés, contraints et muselés.

\section{NOTES}

1. Gustave Flaubert, Lettre du 19 août 1872 à Mme Roger des Genettes.

2. Gustave Flaubert, Bouvard et Pécuchet. Dictionnaire des idées reçues, édition avec dossier, présentée par Stéphanie Dord-Crouslé, « Garnier-Flammarion », Flammarion, Paris, 1999. Toutes les références de pages renvoient à cette édition et sont indiquées, entre parenthèses, après chaque citation.

Arts et Savoirs, 1 | 2012 
3. Jacques Neefs, «La Nuit de Noël. Bouvard et Pécuchet », Mythes et Religions 2, textes réunis par Bernard Masson, Paris, Lettres Modernes Minard, « Gustave Flaubert, 3 », 1988, p. 35-61

4. Sur le principe du Même comme procédé comique, on pourra consulter l'article de Niklas Bender dans ce même numéro. Voir Niklas Bender, "Bouvard et Pécuchet : une mise en scène comique des savoirs ", Bouvard et Pécuchet : la fiction des savoirs, textes réunis par G. Séginger, revue en ligne Arts et savoirs [ISSN 2258-093X], n 1, février 2012. URL : http://lisaa.univ-mlv.fr/ arts-et-savoirs/

5. Philippe Dufour, Flaubert et le Pignouf. Essai sur la représentation romanesque du langage, «L'Imaginaire du Texte », Presses universitaires de Vincennes, Saint-Denis, 1993.

6. L'ouvrage d'Yvan Leclerc reste sur ce point une référence: Yvan Leclerc, La Spirale et le Monument. Essai sur Bouvard et Pécuchet de Gustave Flaubert, «Présences critiques », Paris, SEDES, 1988.

7. Gustave Flaubert, Lettre du 15 avril 1875 à Mme Roger des Genettes.

8. Ce sont les grandes articulations du roman évoquées dans les scénarios (ms gg 10) et dans la Correspondance.

9. Molière, Tartuffe, I, 5.

10. Oswald Ducrot et al., "D'ailleurs ou la logique du camelot», Les Mots du discours, "Le Sens commun », Les Éditions de Minuit, Paris, 1980.

11. Philippe Dufour, Flaubert et le Pignouf, op. cit., p. 117.

12. Oswald Ducrot et al., « D'ailleurs ou la logique du camelot », op. cit., p. 193-232.

13. Ibid., p. 197.

14. Ibid., p. 223.

15. Ibid., p. 206.

16. «Le locuteur prétend viser une conclusion $r$, il donne pour cette conclusion l'argument $P$ qui la justifie. Et, dans un second mouvement discursif, il ajoute un argument $Q$, allant dans le même sens que $P$. Dans la mesure où $P$ tout seul devait déjà conduire à $r, Q$ est ainsi présenté comme n'étant pas nécessaire pour l'argumentation. Le locuteur prétend donc ne pas utiliser $Q$ mais seulement l'évoquer (en d'autres termes, tout en présentant $Q$ comme un argument, il prétend ne pas argumenter à partir de $Q$ ) » (Oswald Ducrot et al., « D'ailleurs ou la logique du camelot », op. cit ., p. 195). L'argument introduit par d'ailleurs n'a donc pas, en soi, valeur de preuve ; ce n'est pas sa nature mais sa présence qui vise à garantir la cohérence du raisonnement qui le précède.

17. Philippe Dufour, Flaubert et le Pignouf, op. cit., p. 25.

18. Ibid., p. 27.

19. Oswald Ducrot, «Analyses pragmatiques ", Communications, $n^{\circ} 32$, Seuil, Paris, 1980, p. 14. Les analyses d'Antoine Culioli sont complémentaires: «mais indique que l'on distingue, par construction, deux zones de validation, telles qu'il existe une relation d'altérité entre les deux zones (altérité signifie qu'il y a différenciation entre les deux zones, qu'il s'agisse d'altérité qualitative ou modale, ou encore d'altérité dans l'orientation: Intérieur vers Centre attracteur, Intérieur vers Extérieur, donc à la Frontière, passage de l'Intérieur à l'Extérieur). À l'invariance (altérité) correspond une variation complexe, liée aux modes et aux domaines d'application de l'altérité. D'où le phénomène de discontinuité abrupte [...]. On marque une position en rupture avec ce que l'on attendait, devant un surgissement qui casse la suite lisse des événements ordinaires. ", Antoine Culioli, « Non mais, des fois! ", Pour une linguistique de l'énonciation. Tome 3. Domaine notionnel, Ophrys, Paris, 1999, p. 138.

20. Jean-Claude Anscombre, Oswald Ducrot, «Deux mais en français? ", Lingua, n 43, 1977, p. 23-40.

21. Anne Herschberg Pierrot, "Effets de voix dans Madame Bovary", Modern Language Notes, Madame Bovary. The Novel as a Modern Art, special editor Jacques Neefs, Vol. 122, $n^{\circ} 4$, The Johns Hopkins Press, Baltimore, 2007, p. 713-734. 
22. Juliette Grange, «Les deux colonnes », Bouvard et Pécuchet centenaires. 1881-1981, textes présentés par Dominique-Gilbert Laporte, Ornicar ?, Paris, 1981.

23. Philippe Dufour, Flaubert et le Pignouf, op. cit., p. 82.

24. Ibid., p. 81. Ainsi «le dithyrambe » et « la diatribe » seraient plus efficaces, plus persuasifs, à défaut d'être plus convaincants, qu'une démonstration rigoureuse. La phrase de La Bruyère est extraite des Caractères, Paris, « Folio », Gallimard, 1979, p. 102.

25. Ibid., p. 116.

26. Ibid.

27. Norioki Sugaya propose dans ce même numéro l'étude du dossier génétique de cet épisode et montre comment, au fil des récritures, s'opère le choix de la formule la plus catégorique pour clore le débat, le «Qu'importe» de Pécuchet étant repris et redoublé de l'impératif démissionnaire de Vaucorbeil. Voir Norioki Sugaya, «La poétique documentaire de Bouvard et Pécuchet. Visibilité des savoirs et arrangement scénarique », Bouvard et Pécuchet : la fiction des savoirs, textes réunis par G. Séginger, revue en ligne Arts et savoirs, [ISSN 2258-093X], n 1, février 2012. URL : http://lisaa.univ-mlv.fr/arts-et-savoirs, p. 4-6.

28. Oswald Ducrot et al., Les Mots du discours, op.cit., p.47-49 et «Esquisse d'une théorie polyphonique de l'énonciation », Le Dire et le Dit, «Propositions », Les Éditions de Minuit, Paris, 1984.

29. Oswald Ducrot et al., Les Mots du discours, op. cit., p. 48.

30. Philippe Dufour, Flaubert et le Pignouf, op. cit., p. 117.

31. Ibid., p. 119.

32. Ibid., p. 117.

33. Ibid., p. 118.

34. Ibid., p. 120.

35. Jacques Neefs, «Bouvard et Pécuchet, la prose des savoirs ", Théorie, Littérature, Épistémologie, $\mathrm{n}$ - 10, Épistémocritique et cognition 1, Presses universitaires de Vincennes, Saint-Denis, 1992.

36. «Le paradoxe [...] est de faire se rencontrer dans la simple succession du relevé, l'extrême singularité d'une humeur [...] et la tranche commune d'une époque, de ses habitudes, de ses goûts, de ses modes, de ses tics [...]. Se faire sujet du discours anonyme d'un temps et d'une culture, être traversé par ce commun envahissant qui fait le temps précaire de l'opinion et de la mode, pour l'exposer et s'en défaire, pour lui donner une forme qui sera celle de la durée et de l'œuvre, c'est bien toujours l'entreprise de Flaubert romancier qui s'exerce là, mais de manière plus abrupte. ", Jacques Neefs, « Noter, classer, briser, montrer, les dossiers de Bouvard et Pécuchet ", Penser, classer, écrire. De Pascal à Perec, études réunies et présentées par Béatrice Didier et Jacques Neefs, « Manuscrits Modernes », Presses Universitaires de Vincennes, Saint-Denis, 1990, p. 89.

37. Norioki Sugaya, «L'impossible savoir médical : sur les notes médicales de Bouvard et Pécuchet ", Revue de langue et littérature françaises, $n^{\circ} 17$, Société de langue et littérature françaises de l'université de Tokyo, 1998, p. 159.

INDEX

Mots-clés : savoir, pouvoir, argumentation 
AUTEUR

FLORENCE PELLEGRINI

Université Bordeaux Montaigne - EA 4195 Telem 